\title{
Non-small cell neuroendocrine carcinoma of the ovary in a BRCA2-germline mutation carrier: A case report and brief review of the literature
}

\author{
NATALIE HEROLD ${ }^{1}$, BARBARA WAPPENSCHMIDT ${ }^{1}$, BIRGID MARKIEFKA ${ }^{2}$, KATHARINA KEUPP ${ }^{1}$, \\ SANDRA KRÖBER $^{1}$, ERIC HAHNEN ${ }^{1}$, RITA SCHMUTZLER ${ }^{1}$ and KERSTIN RHIEM ${ }^{1}$ \\ ${ }^{1}$ Center for Familial Breast and Ovarian Cancer, Center for Integrated Oncology, Medical Faculty; \\ ${ }^{2}$ Institute for Pathology, University Hospital of Cologne, D-50931 Cologne, Germany
}

Received March 6, 2017; Accepted June 9, 2017

DOI: $10.3892 / \mathrm{ol} .2018 .7836$

\begin{abstract}
Non-small cell neuroendocrine carcinomas (NSCNEC) account for $2 \%$ of gynecological cancer cases and are associated with a poor prognosis due to delayed diagnosis and aggressive tumor behavior. BRCA2-associated ovarian carcinomas predominantly possess a high-grade serous phenotype, which respond to platinum and targeted therapy with PARP inhibitors. Presented here is the case of an adult patient with NSCNEC of the ovaries associated with a deleterious BRCA2 germline mutation. The pathogenic mutation was also confirmed on the somatic level, while the wild-type allele had a high variant fraction, suggesting loss of heterozygosity. To the best of our knowledge, this is the first report of an adult BRCA2 germline mutation carrier with the rare NSCNEC of the ovary phenotype. Therefore, ovarian cancer patients with histological subtypes other than high-grade serous carcinomas should be tested for $B R C A 1 / 2$ mutations, as they may benefit from targeted therapy with poly (ADP-ribose) polymerase inhibitors.
\end{abstract}

\section{Introduction}

BRCA2 mutation carriers have an increased risk (11-17\%) of developing ovarian cancer $(1,2)$. The typical phenotype of $B R C A 2$-associated ovarian cancer is a high-grade serous papillary carcinoma (3-6). The tumor suppressor genes $B R C A 1 / 2$ serve a pivotal role in the process of homologous recombination (HR) repair of double-strand DNA breaks $(7,8)$. The more promising prognosis of $B R C A$-associated ovarian carcinomas is considered to be due to favorable responses to a DNA-damaging treatment

Correspondence to: Dr Rita Schmutzler, Center for Familial Breast and Ovarian Cancer, Center for Integrated Oncology, Medical Faculty, University Hospital of Cologne, 34 Kerpener Street, D-50931 Cologne, Germany

E-mail: rita.schmutzler@uk-koeln.de

Key words: BRCA2, neuroendocrine carcinoma, ovarian carcinoma, mutation, poly (ADP-ribose) polymerase inhibitor regimen containing, such as with carboplatin (9). Another targeted therapeutic option for patients with $B R C A$-associated high grade serous ovarian cancer is the poly (ADP-ribose) polymerase (PARP) inhibitor olaparib, which has recently been approved by the US Food and Drug Administration and the European Medicines Agency. PARP-1 is a key enzyme involved in the repair of DNA single-strand breaks. In the absence of PARP activity, these lesions are converted into double strand breaks. $B R C A$-deficient cells are unable to repair this damage by $\mathrm{HR}$ and accumulate unrepaired breaks, resulting in apoptosis (10-12).

Tumors of the diffuse neuroendocrine cell system may arise in the vulva, vagina, cervix, endometrium or ovary (13), and account for only $2 \%$ of gynecologic cancer cases (13). Non-small cell neuroendocrine carcinomas (NSCNEC) of the ovary, also known as large cell neuroendocrine carcinomas, are rare. To date, the Society of Gynecologic Oncology has reported only 35 cases (14). NSCNEC is associated with a poor prognosis due to late presentation and aggressive behavior (15). Hitherto, only one case of a poorly differentiated mediastinal neuroendocrine carcinoma in a pediatric patient with a germline mutation in $B R C A 2$ has been published with a loss of heterozygosity $(\mathrm{LOH})$ involving the normal allele in tumor tissue (16).

Herein, a case of an adult patient with a background of familial breast cancer suffering from a rare phenotype of NSCNEC of the ovary is presented. Germline and tumor mutation analysis were performed using the TruRisk ${ }^{\circledR}$ gene panel. A deleterious germline mutation in the BRCA2 gene was subsequently diagnosed in both germline and tumor tissue, while the wild-type allele was rarely detected in tumor tissue, suggesting LOH. Furthermore, a somatic mutation in TP53 was detected, which is typical for $B R C A$-associated tumors. The current case report also reveals that histologic subtypes other than high-grade serous ovarian cancer are associated with $B R C A 1 / 2$ mutations in germline and tumor tissue, and thus may benefit from PARP inhibitor therapy.

\section{Case report}

A post-menopausal, 75-year-old female patient was diagnosed with a solid tumor mass of the right ovary of $7 \mathrm{~cm}$ in 
December 2013 at the Department of Gynaecology of the Vinzenz Pallotti Hospital in Bergisch Gladbach, Germany. According to the medical history, the patient (gravida 2, para 2) underwent a vaginal hysterectomy at the age of 37 , and an appendectomy later on. Pre-operative tumor markers were as follows: Cancer antigen (CA)-125 was elevated to $63.4 \mathrm{U} / \mathrm{ml}$ (normal <35 U/ml); carcinoembryonic antigen, CA 19-9 and CA 72-4 were within the normal range. Laparoscopy revealed a suspect tumor. Initial laparoscopy was converted to a debulking surgery according to S3-guideline on the diagnostics, therapy and follow-up of malignant ovarian tumors (17). Abdominal bilateral salpingo-oophorectomy, omentectomy, and deperitonealisation of the pelvis were performed, as well as a pelvic and paraaortal lymphonodectomy. An atypical liver resection was performed due to metastases. Ethical approval was provided by the Ethics Committee of the University of Cologne (Cologne, Germany).

The tumor measured 13x7x6 cm and weighed $290 \mathrm{~g}$. The final histological analysis revealed an NSCNEC of the ovary. According to the International Federation of Gynecology and Obstetrics and the NCCN Guidelines, the tumor is classified as pT3, pN1, M1, FIGO Stage IV $(18,19)$. The diagnosis of the local pathologist was verified by a reference pathologist of the Institute of Pathology Marburg/Wiesbaden. Immunohistochemical staining was positive for synaptophysin and CDX2; chromogranin A was partially positive; the cells were negative for cytokeratin (CK) 20 and thyroidal transcription factor 1. Examination of proliferative activity using monoclonal antibody MIB-1 (antigen Ki-67 index) revealed nuclear positivity in $~ 90 \%$ of tumor cells and dot-like expression patterns of CK 8/18. Immunohistochemical staining confirmed positive stains for synaptophysin and CD56 (Fig. 1).

Postoperative gastroscopy and colonoscopy, as well as computer tomography of the thorax and abdomen, revealed no evidence for a second primary cancer and no further distant metastases than in the omentum and in the liver, which had been successfully resected. After surgery, the patient underwent chemotherapy with two cycles of etoposide and carboplatin, followed by paclitaxel and carboplatin. The healing process was prolonged by wound healing complications treated with vacuum sealing, and a subileus. The patient was treated for neutropenia and nosocomial pneumonia. Until December 2016, there were no signs of recurrent disease.

The patient had a familial history of breast cancer according to the inclusion criteria of the GC-HBOC (20). A three-generation pedigree was drawn and phenotypes were documented. The patient's mother and three aunts were diagnosed with breast cancer at the ages of 62, 60,79 and 61 years, respectively. The maternal female cousin was diagnosed with breast cancer at the age of 57. As the person with the earliest onset of breast cancer, she was considered the index case within the family. The only uncle was diagnosed with prostate cancer at the age of 65 as well as one of the patient's three brothers (Fig. 2).

The pattern of breast and prostate cancer suggested the presence of a dominantly inherited cancer susceptibility gene. Following genetic counseling and the provision of informed consent, the female cousin, as the index case, and the primary patient of the present study agreed to genetic testing. Written informed consent was obtained from the patients in
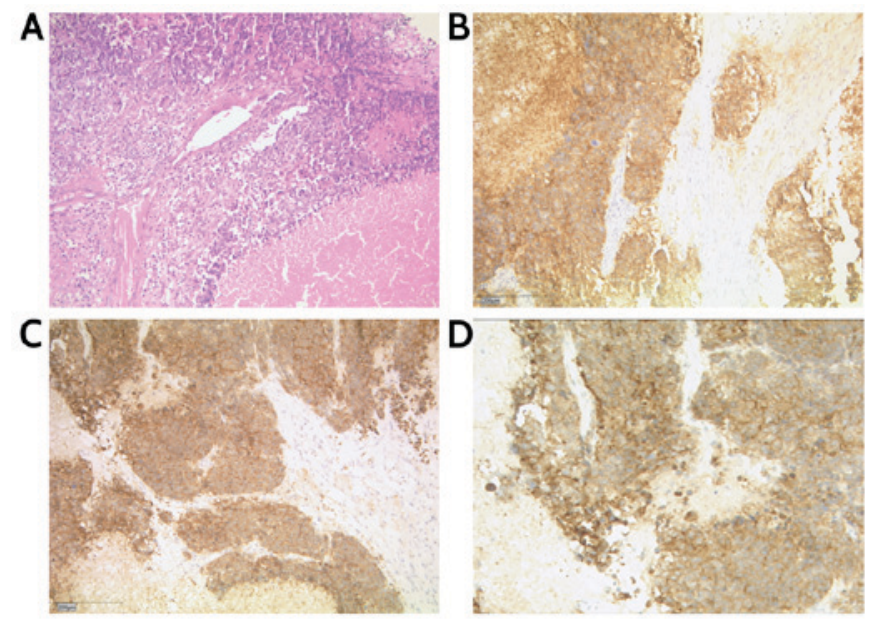

Figure 1. Non-small cell neuroendocrine carcinoma of the ovary. (A) Hematoxylin and eosin staining, magnification x100. (B) Immunohistochemical staining: CD56, magnification x100. (C and D) Synaptophysin, magnification $\mathrm{x} 100$.

accordance with the Declaration of Helsinki. Blood samples were collected within the Center for Familial Breast and Ovarian Cancer, University of Cologne (Cologne, Germany); the local pathologist at the Institute of Pathology of the Vinzenz Pallotti Hospital in Bergisch Gladbach, where initial surgery was performed, provided tumor tissue. Genomic DNA was isolated from venous blood samples and tumor tissues. DNA extraction from blood samples was performed by an automated high-throughput nucleic acid isolation method based on proprietary M-PVA magnetic bead technology $\left(\right.$ Chemagic $^{\mathrm{TM}}$ MSM I instrument; Chemagen; PerkinElmer, Inc., Baesweiler, Germany) according to the manufacturer's instructions. DNA was quantified using a NanoDrop spectrophotometer ND-1000 and NanoDrop 1003.7 software (NanoDrop; Thermo Fisher Scientific, Inc., Wilmington, DE, USA). DNA extraction from formalin-fixed, paraffin-embedded (FFPE) material was performed with a Maxwell ${ }^{\circledR} 16$ FFPE Plus Tissue LEV DNA Purification kit (Promega Corporation, Madison, WI, USA) on the Maxwell ${ }^{\circledR} 16$ (Promega Corporation). According to the manufacturer's instructions, $10 \mu \mathrm{m}$ thick tissue sections were cut from the FFPE blocks. Following deparaffinization, tumor areas were macrodissected from the unstained slides using a sterile scalpel. A previously marked hematoxylin and eosin stained slide served as the reference. The samples were screened for gross genomic aberrations in the BRCAl/2 genes by Multiplex Ligation-dependent Probe Amplification (MLPA) using the SALSA ${ }^{\circledR}$ MLPA $^{\circledR}$ probe mixes P002 (BRCA1) and P045 (BRCA2; MRC-Holland, Amsterdam, The Netherlands), according to the manufacturer's protocol. Data were analyzed using the Coffalyser.Net v.140429.1057 software (MRC-Holland). For next generation sequencing, a customer-tailored multigene panel (TruRisk ${ }^{\circledR}$; Agilent Technologies, Inc., Santa Clara, CA, USA) was employed, covering BRCA1, BRCA2 and further non-BRCA1/2 cancer predisposition genes, using the SureSelect XT Target Enrichment for Illumina Paired-End Multiplexed Sequencing protocol according to the manufacturer's instructions (Agilent Technologies, Inc.) optimized for 200 ng genomic tumor DNA and $3 \mu \mathrm{g}$ genomic blood DNA. Sequencing was performed on a 


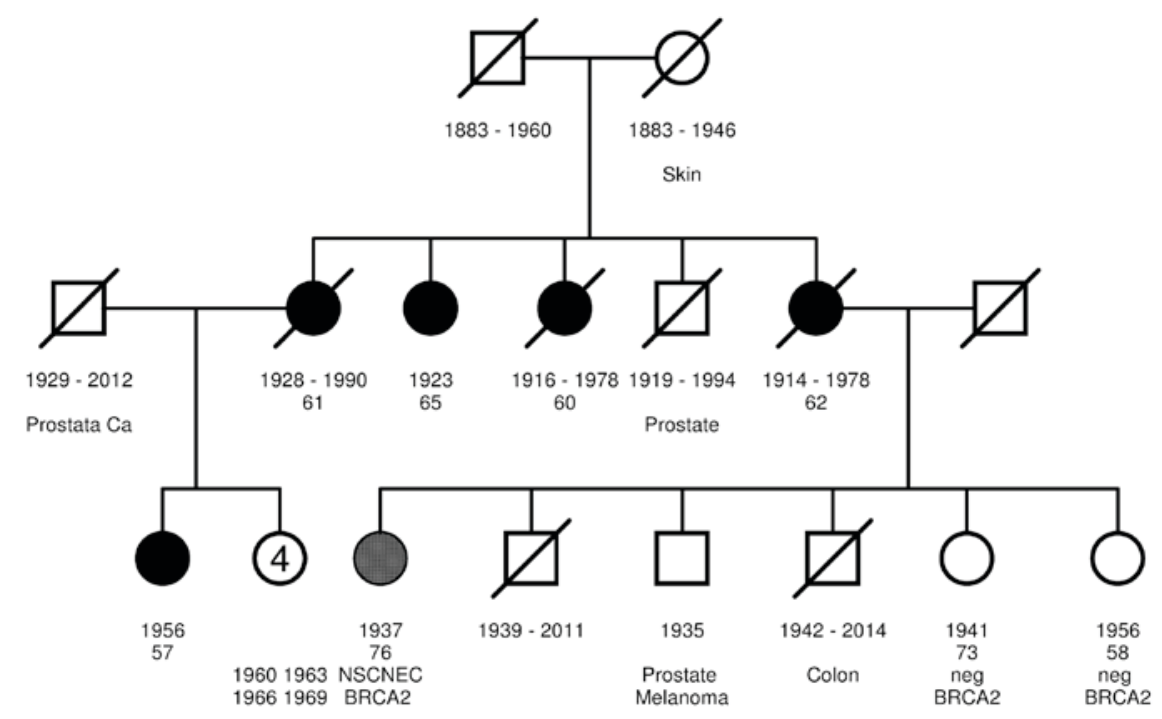

Figure 2. Three-generation pedigree. Square, male; circle, female; white-filled square/circle, no relevant disease; strikethrough, deceased; filled grey circle, patient with NSCNEC; filled black circle, patient with breast cancer. Year of birth and age of tumor diagnosis are provided. Predictive negative genetic test results are provided.

MiSeq (tumor DNA) and a HiSeq 4000 (blood DNA; Illumina, Inc., San Diego, CA, USA). Bioinformatic analyses were carried out using the Sequence Pilot software version 4.2.2 (JSI Medical Systems GmbH, Ettenheim, Germany). Deleterious mutations identified via this approach were classified according to the IARC system (21) then verified using standard Sanger sequencing with 25-50 ng DNA using the BigDye ${ }^{\circledR}$ Terminator method (ABI PRISM BigDye Terminator v1.1 Ready Reaction Cycle Sequencing kit; Applied Biosystems; Thermo Fisher Scientific, Inc., Waltham, MA, USA) on an ABI 3500xL sequencer (Applied Biosystems; Thermo Fisher Scientific, Inc.), according to manufacturer's instructions.

The following primers were used (supplied by Metabion international AG Metabion GmbH; Planegg/Steinkirchen, Germany): BRCA2 forward, 5'-GCTAATAGATGCCTAAG CCC-3' and reverse, 5'-GAAACCTTAACCATACTGCCG-3'; and TP53 forward, 5'-CACTTGTGCCCTGACTTTCAAC TCT-3' and reverse, 5'-GAGAAAGCCCCCCTACTGCTCA-3'

\section{Results}

In the current case, a heterozygous germline mutation, $B R C A 2$ exon 17, c.7976G >A, p.(Arg2659Lys), was identified in the family's index case, the female cousin diagnosed with breast cancer at the age of 57 . The mutation was classified as $>99 \%$ pathogenic (class 5) according to the IARC system (21) and functional analyses of the Evidence-based Network for the Interpretation of Germline Mutant Alleles consortium, which comprises genetic data of the GC-HBOC database $(20,22)$. The patient with NSCNEC was identified to be a heterozygous carrier of the family's pathogenic germline mutation in BRCA2. No further pathogenic germline mutations were identified using the TruRisk ${ }^{\circledR}$ genepanel.

Somatic mutation analysis in our patient revealed a deleterious somatic mutation in TP53, c.600delT,p.(Leu201Cysfs*46). The variant fraction of the known germline mutation in $B R C A 2$ exhibited a marked increase from $47 \%$ (412/876 reads) in germline to $83 \%$ (198/238 reads) in tumor tissue, suggesting a LOH.
A

TAAAATACAGGCAAC

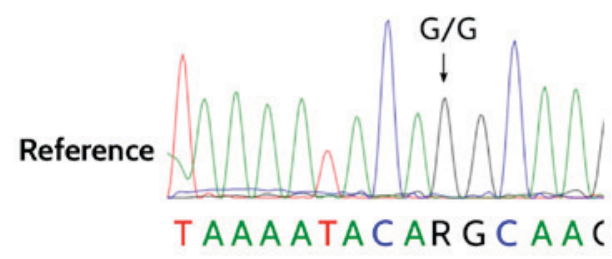

B

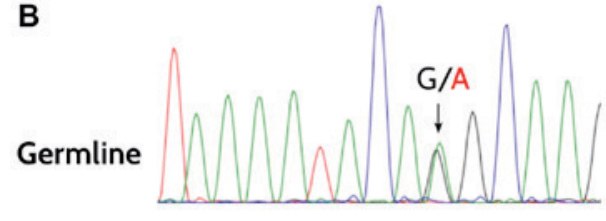

TA AAATACAAG CAAG

C

Tumor

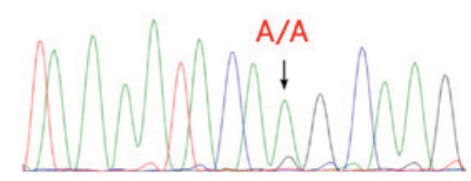

Figure 3. Heterozygous germline mutation in BRCA2 exon 17, c.7976G $>A$, p.(Arg2659Lys). (A-C) Demonstration of the loss of heterozygosity analysis in tumor cells (C), the heterozygous germline mutation (B) and a wild-type reference of a healthy predicted-negative relative (A).

The subsequent Sanger sequencing of the tumor-derived DNA confirmed the loss of the wild-type allele. Predictive testing and genetic counseling regarding preventive measures were offered to the male and female relatives of the patient. Written informed consent was obtained from two healthy sisters in accordance with the Declaration of Helsinki, they turned out to be predictive negative for the germline mutation (Fig. 3).

\section{Discussion}

The case presented here is, to the best of our knowledge, the first case of an adult NSCNEC of the ovary associated with a 
deleterious germline mutation in the $B R C A 2$ gene. The patient is a 75-year-old female who underwent surgery and adjuvant chemotherapy for a unilateral NSCNEC of the ovary without surface epithelial components. The last follow up appointment was in December 2016 and the patient remained alive without any evidence of recurrent disease. She is currently being monitored within the regular follow up schedule of ovarian carcinomas according to the S3-guideline on the diagnostics, therapy and follow-up of malignant ovarian tumors (17).

Due to a low prevalence and poor prognosis, there is currently only limited knowledge regarding the clinical management of this aggressive tumor, which is associated with high mortality despite extensive surgery and adjuvant chemotherapy. BRCA1/BRCA2 mutation analysis in germline and tumor tissue may provide additional targeted therapeutic options, e.g., with PARP inhibitors.

\section{References}

1. Wooster R, Neuhausen SL, Mangion J, Quirk Y, Ford D, Collins N, Nguyen K, Seal S, Tran T, Averill D, et al: Localization of a breast cancer susceptibility gene, BRCA2, to chromosome 13q12-13. Science 265: 2088-2090, 1994.

2. Antoniou A, Pharoah PD, Narod S, Risch HA, Eyfjord JE, Hopper JL, Loman N, Olsson H, Johannsson O, Borg A, et al: Average risks of breast and ovarian cancer associated with BRCA1 or BRCA 2 mutations detected in case series unselected for family history: A combined analysis of 22 studies. Am J Hum Genet 72: 1117-1130, 2003.

3. Lakhani SR, Manek S, Penault-Llorca F, Flanagan A, Arnout L, Merrett S, McGuffog L, Steele D, Devilee P, Klijn JG, et al: Pathology of ovarian cancers in BRCA1 and BRCA2 carriers Clin Cancer Res 10: 2473-2481, 2004.

4. Soegaard M, Kjær SK, Cox M, Wozniak E, Høgdall E, Høgdall C, Blaakaer J, Jacobs IJ, Gayther SA and Ramus SJ: BRCA1 and BRCA2 mutation prevalence and clinical characteristics of a population-based series of ovarian cancer cases from denmark. Clin Cancer Res 14: 3761-3767, 2008.

5. Chetrit A, Hirsh-Yechezkel G, Ben-David Y,Lubin F, Friedman E and Sadetzki S: Effect of BRCA1/2 mutations on long-term survival of patients with invasive ovarian cancer: The national Israeli study of ovarian cancer. J Clin Oncol 26: 20-25, 2008.

6. Cass I, Baldwin RL, Varkey T, Moslehi R, Narod SA and Karlan BY: Improved survival in women with BRCA associated ovarian carcinoma. Cancer 97: 2187-2195, 2003.

7. Moynahan ME, Chiu JW, Koller BH and Jasin M: BRCA1 controls homology-directed DNA repair. Mol Cell 4: 511-518, 1999.

8. Moynahan ME, Pierce AJ and Jasin M: BRCA2 Is required for homology-directed repair of chromosomal breaks. Mol Cell 7: 263-272, 2001.

9. Bolton KL, Chevenvix-Trench G, Goh C, Sadetzki S, Ramus SJ, Karlan BY, Lambrechts D, Despierre E, Barrowdale D, McGuffog L, et al: Association between BRCA1 and BRCA2 mutations and survival in women with invasive epithelial ovarian cancer. JAMA 307: 382-390, 2012.
10. Fong PC, Boss DS, Yap TA, Tutt A, Wu P, Mergui-Roelvink M, Mortimer P, Swaisland H, Lau A, O'Connor MJ, et al: Inhibition of poly(ADP-ribose) polymerase in tumors from BRCA mutation carriers. N Engl J Med 361: 123-134, 2009.

11. Audeh MW, Carmichael J, Penson RT, Friedlander M, Powell B, Bell-McGuinn KM, Scott C, Weitzel JN, Oaknin A, Loman N, et al: Oral poly(ADP-ribose) polymerase inhibitor olaparib in patients with BRCA1 or BRCA2 mutations and recurrent ovarian cancer: A proof-of-concept trial. Lancet 376: 245-251, 2010.

12. Nijman SM: Synthetic lethality: General principles, utility and detection using genetic screens in human cells. FEBS Lett 585: $1-6,2011$

13. Rouzbahman M and Clarke B: Neuroendocrine tumors of the gynecologic tract: Select topics. Semin Diagn Pathol 30: 224-233, 2013.

14. Gardner GJ, Reidy-Lagunes D and Gehrig PA: Neuroendocrine tumors of the gynecologic tract: A Society of Gynecologic Oncology (SGO) clinical document. Gynecol Oncol 122: 190-198, 2011.

15. Taube ET, Denkert C, Pietzner K, Dietel M, Sehouli J and Darb-Esfahani S. Prognostic impact of neuroendocrine differentiation in high-grade serous ovarian carcinoma. Virchows Arch 466: 333-342, 2015.

16. Bhatla T, Dandekar S, Lu BY, Wang J, Han E, Bitterman D, Jones CL, Evensen NA, Magid M, Meyer JA and Carroll WL: Genomic characterization of poorly differentiated neuroendocrine carcinoma in a pediatric patient. J Pediatr Hematol Oncol 38, e21-e25, 2016.

17. Wagner U, Harter P, Hilpert F, Mahner S, Reuß A, du Bois A, Petru E, Meier W, Ortner P, König K, et al: S3-Guideline on Diagnostics, Therapy and Follow-up of Malignant Ovarian Tumors: Short version 1.0- AWMF registration number: 032/035OL, June 2013. Geburtshilfe Frauenheilkd 73: 874-889, 2013.

18. Prat J; FIGO Committee on Gynecologic Oncology: FIGO's staging classification for cancer of the ovary, fallopian tube, and peritoneum: Abridged republication. J Gynecol Oncol 26: 87-89, 2015.

19. National Comprehensive Cancer Network. NCCN Clinical Practise Guidelines in Oncology: (NCCN) Ovarian cvancer including fallopian tube cancer and primary peritoneal cancer. Version 1, 2015

20. Meindl A, Ditsch N, Kast K, Rhiem K and Schmutzler RK: Hereditary breast and ovarian cancer: New genes, new treatments, new concepts. Dtsch Arztebl Int 108: 323-330, 2011.

21. Plon SE, Eccles DM, Easton D, Foulkes WD, Genuardi M, Greenblatt MS, Hogervorst FB, Hoogerbrugge N, Sprudle AB and Tavtigian SV; Tavtigian SV; IARC Unclassified Genetic Variants Working Group: Sequence variant classification and reporting: Recommendations for improving the interpretation of cancer susceptibility genetic test results. Hum Mutat 29: 1282-1291, 2008.

22. Easton DF, Pharoah PD, Antoniou AC, Tischkowitz M, Tavtigian SV, Nathanson KL, Devilee P, Meindl A, Couch FJ, Southey M, et al: Gene-panel sequencing and the prediction of breast-cancer risk. N Engl J Med 372: 2243-2257, 2015.

This work is licensed under a Creative Commons Attribution-NonCommercial-NoDerivatives 4.0 International (CC BY-NC-ND 4.0) License. 